J. Lake Sci.(湖泊科学), 2017, 29(2): 490-497

DOI 10. 18307/2017. 0225

(c) 2017 by Journal of Lake Sciences

\title{
基于梯度变换的浅水湖泊围网区遥感提取算法
}

\author{
黄 帅 $^{1}$, 宋开宏 $^{1 * *}$, 罗菊花 $^{2}$, 赵晋陵 $^{1}$, 马荣华 ${ }^{2}$ \\ ( 1 : 安徽大学计算智能与信号处理教育部重点实验室, 合肥 230039) \\ (2: 中国科学院流域地理学重点实验室中国科学院南京地理与湖泊研究所,南京 210008)
}

摘 要: 获取并掌握浅水湖泊围网养殖区域的时空分布信息对合理规划围网养殖进而提升湖泊水质具有重要意义. 本文 以长江下游典型的围网养殖浅水湖泊一一阳澄湖作为研究区, 利用资源三号 (ZY-3) 高分遥感影像, 针对围网区与非围网 区的光谱空间变化特征, 采用梯度变换方法, 尝试提出一种浅水湖泊围网区的遥感提取算法; 并以人工解译结果作为参 考, 对提取结果进行验证. 研究结果发现该算法对浅水湖泊围网养殖区的提取精度为 $90.66 \%$, 可进一步用于开展长时序 的浅水湖泊围网区动态变化研究,进而为湖泊环境的政府部门制定湖泊水质提升和围网区合理规划政策提供决策依据.

关键词: 浅水湖泊; 围网养殖区;梯度变换;卫星遥感;阳澄湖

\section{A remote sensing extraction algorithm of enclosure culture area in shallow lakes based on gradient transform}

HUANG Shuai ${ }^{1}$, SONG Kaihong ${ }^{1 * *}$, LUO Juhua ${ }^{2}$, ZHAO Jinling $^{1} \&$ MA Ronghua ${ }^{2}$

(1: Key Laboratory of Intelligent Computing \& Signal Processing, Ministry of Education, Anhui University, Hefei 230039, P.R.China)

(2: Key Laboratory of Watershed Geographic Sciences, Nanjing Institute of Geography and Limnology, Chinese Academy of Sciences, Nanjing 210008, P.R.China)

Abstract: Mastering the tempo-spatial distribution information of enclosure culture areas is useful for a scientific planning of enclosure culture areas of shallow lakes and making effective measurements to improve water quality. This study took Lake Yangcheng as a study area, which is a typical enclosure culture area in the lower reaches of Yangtze River. An extraction algorithm of remote sensing images is proposed through the gradient transformation of remote sensing data based on the differences between enclosure and non-enclosure areas in spectral space. A high-resolution ZY-3 image was used to acquire the spatial distribution of enclosure culture areas in shallow lakes. The enclosure culture areas extracted using the proposed extraction algorithm and the manual visual interpretation were compared to evaluate the classification accuracy. The results show that the overall classification accuracy reached to $90.66 \%$. The proposed method could be used to monitor the dynamic changes of enclosure culture areas in shallow lakes based on the ZY-3 images.

Keywords: Shallow lakes; enclosure culture area; gradient transformation; satellite remote sensing; Lake Yangcheng

随着社会经济的快速发展, 我国的水产渔业也稳步向前, 逐渐从过度消耗天然渔业到人工放养再到围 网养殖 ${ }^{[1]}$. 近 20 年来, 长江流域的浅水湖泊围网养殖业快速扩张, 极大地增加了经济效益, 但超高密度的围 网养殖也对湖泊生态造成了严重破坏.一方面,围网养殖区中的围网会阻隔水流, 减缓污染物的扩散速度, 且投放的饵料增加氮、磷等浓度, 加剧了浅水湖泊的内源污染 ${ }^{[2]}$; 另一方面, 养殖的鱼蟹消耗甚至破坏了水 生植物, 降低湖泊的自净能力, 打破了湖泊的生态平衡 ${ }^{[3]}$. 在此背景下, 合理科学地规划并控制湖泊围网已 被湖泊管理部门列为湖泊水质提升和优化的重点工作, 而获取并掌握湖泊围网养殖的时空分布信息是湖泊

* 国家自然科学基金项目 (41301375) 和苏州市阳澄湖生态系统优化提升研究基金项目 (SZLHZ 2014-G-003) 联合资 助. 2016-04-13 收稿;2016-06-02 收修改稿.黄帅 (1992 ), 男,硕士研究生;E-mail : adamw300462@ 163.com.

** 通信作者; E-mail:sk_hong@ sina.com. 
生态管理部门在科学管理围网养殖区时制定措施的依据.

传统的围网养殖区空间分布及其面积的获取方法即人工实地调查测量法, 该方法时间周期长, 人力投 人大. 遥感技术由于其具有实时、大范围、动态和客观等优势, 而逐渐成为湖泊围网养殖区监测和湖泊水环 境调查的主流方法. 沈芳等 ${ }^{[4]}$ 采用人工目视解译遥感影像后数字化的方法, 提取了滆湖和洮湖围网养殖区. 人工目视解译虽具有较高的精度, 但仍然费时费力, 很难批量自动提取. 为克服该方法无法批量提取的缺 陷,近年来,学者们先后基于各种遥感数据探讨了围网区的自动提取方法. 如,马艳娟等 ${ }^{[5]}$ 基于 ASTER 数据 直接利用光谱信息提取了烟台附近的近海围网养殖区; 朱长明等 ${ }^{[6]}$ 基于高分影像通过纹理信息提取了连云 港附近的近海围网养殖区; 卢业伟等 ${ }^{[7]}$ 基于 RAPIDEYE 数据和纹理信息提取了澳门附近的近海围网养殖 区. 但针对浅水湖泊的围网养殖区自动提取方法的研究相对较少,李俊杰等 ${ }^{[8]}$ 基于资源卫星 2 号初步探讨 了如何运用纹理信息提取围网养殖区; 王静等 ${ }^{[9]}$ 基于 Landsat ETM 卫星数据通过对应分析的方法尝试提取 围网养殖区. 以上研究主要集中于使用影像的纹理信息提取围网养殖区,但图像的大多纹理特征计算复杂, 运算量大, 且不同时相的影像往往需要使用不同的纹理特征. 梯度变换作为图像处理和模式识别的常用方 法, 自提出就受到了广泛运用,在此基础上提出的图像处理算法层出不穷. 袁春兰等 ${ }^{[10]}$ 使用 Sobel 算子对用 过 Otsu 算法运算后的红外图像进行梯度变换成功检测到该图像的边缘; 何春华等 ${ }^{[11]}$ 使用自适应权值的 Sobel 算子进行图像的梯度变换检测图像边缘并获得较好结果; 李灿灿等 ${ }^{[12]}$ 使用多方向 Sobel 算子对叶脉图 像进行梯度变换以检测其边缘变化且得到很高的检测精度. 以上基于不同 Sobel 算子的边缘检测研究都表 明了梯度变换可以有效地提取图像边缘, 增强图像细节 ${ }^{[13-14]}$.

本文以长江下游典型的围网养殖湖泊——江苏苏州阳澄湖为研究区, 以资源三号 (ZY-3) 高分影像为数 据源, 利用梯度变换尝试提出一种普适性和准确率较高的围网养殖区遥感提取算法,提取浅水湖泊围网养 殖区,也可为湖泊环境的政府部门制定湖泊水质提升和围网区合理规划政策提供支持.

\section{1 研究区概况与数据获取}

\section{1 研究区概况}

阳澄湖 ( $31^{\circ} 21^{\prime} \sim 31^{\circ} 30^{\prime} \mathrm{N}, 120^{\circ} 39^{\prime} \sim 120^{\circ} 51^{\prime} \mathrm{E}$ ) 地处江苏苏州吴中区与昆山市之间, 是古太湖的残留, 南 北长 $17 \mathrm{~km}$, 东西最大宽度 $8 \mathrm{~km}$, 面积 $119.04 \mathrm{~km}^{2}$, 蓄水量 $1.67 \times 10^{8} \mathrm{~m}^{3}$, 平均水深 $1.4 \mathrm{~m}^{[15]}$. 阳澄湖平均水深 适中, 利于鱼蟹存活, 水产资源十分丰富, 湖中六宝远近闻名, 其中大闸蟹更是驰名中外, 也是较早开展围网 养殖的浅水湖泊之一. 自 1992 年开始, 阳澄湖进行生产性围网养殖, 在 21 世纪初形成围网养殖高峰, 其中 主要以大闸蟹养殖为主.

\section{2 数据搜集}

本文选用的遥感数据源为国产的高分辨率多光谱卫星影像数据——资源三号 $(\mathrm{ZY}-3)$, 影像获取时间是 2015 年 4 月 25 日, 影像多光谱数据空间分辨率为 $5.8 \mathrm{~m}$, 数据记录格式为 GEOTIFF, 产品总共有 4 个波段, 分别是近红外波段 ( $0.77 ~ 0.89 \mu \mathrm{m})$ 、红波段 ( $0.63 \sim 0.69 \mu \mathrm{m})$ 、绿波段 $(0.52 \sim 0.59 \mu \mathrm{m})$ 和蓝波段 $(0.45 \sim 0.52$ $\mu \mathrm{m}$ ). 结合阳澄湖的矢量边界对影像进行裁切 (图 1), 剪切后的研究区影像共计 $3147 * 2736$ 个像元. 考虑到 背景在各波段的灰度值均为 0 ,因此将各波段均为 0 的像元值赋值为 NAN.

\section{2 研究方法与提取结果}

\section{1 方法概述}

典型围网区中围网和自然水体在 ZY-3 影像各波段的灰度值可以看出 (图 2), 围网和自然水体对光谱吸 收有差异,波长最长的近红外波段在围网区的灰度值变化率最大. 因此,可以通过计算近红外波段值的空间 变化率来区分围网和自然水体, 而计算变化率在数学上最常用的工具是梯度. 本文尝试以梯度计算为核心 提出一种新算法提取围网区,具体流程如图 3.

\section{2 梯度变换及优化}

梯度是描述函数变化的矢量,一般用 $\nabla$ 表示, 其模值是函数在最大变化方向上的变化率大小. 二维平 


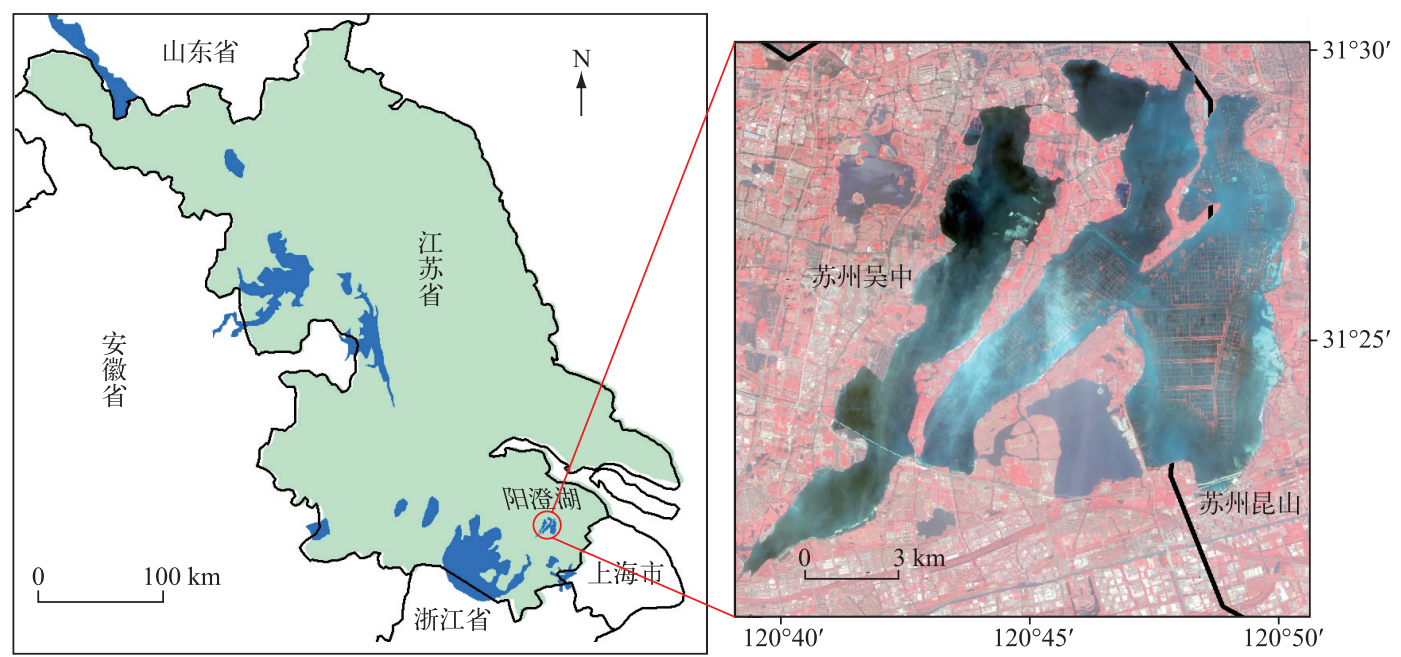

图 1 研究区示意图

Fig.1 Map of study area

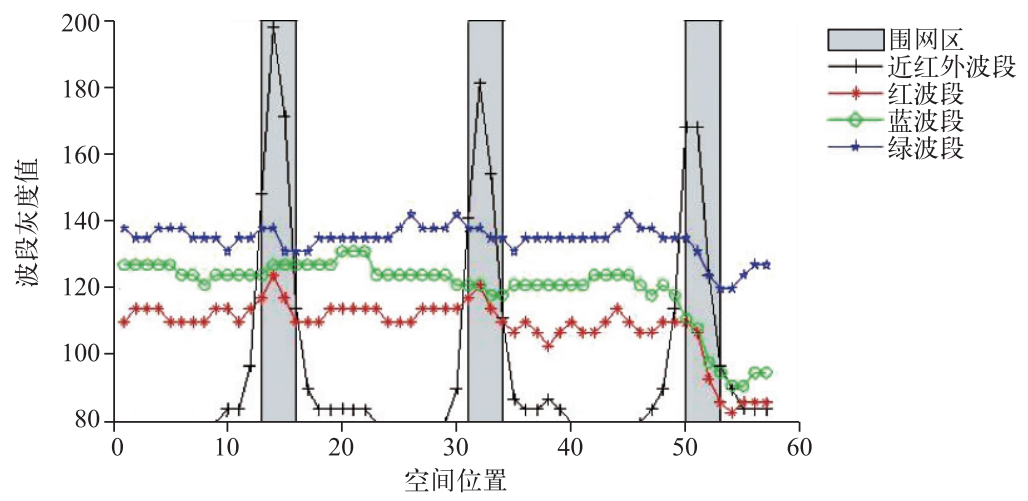

图 2 典型围网区一个样线上各波段的灰度值

Fig.2 Gray values of spectral bandsin typical enclosure culture area

面上,函数 $\varphi$ 的梯度可用列向量 $\nabla \varphi=\left[\begin{array}{l}\frac{\partial \varphi(x, y)}{\partial x} \\ \frac{\partial \varphi(x, y)}{\partial y}\end{array}\right]$ 表示,模值为:
$|\nabla \varphi|=\left[\left(\frac{\partial \varphi(x, y)}{\partial x}\right)^{2}+\left(\frac{\partial \varphi(x, y)}{\partial y}\right)^{2}\right]^{\frac{1}{2}}$

式中, $x$ 表示该点相对于参考点的横向距离, $y$ 表示该点相对于参考点的纵向距离.

实际应用中, 为简化变化率, 即梯度模值的计算, 认为在 $x 、 y$ 变化较小时, 可以用绝对值和近似平方和的 平方根表示 ${ }^{[16]}$,式(1) 也可以表示为:

$$
|\nabla \varphi(x, y)| \approx\left|\frac{\partial \varphi(x, y)}{\partial x}\right|+\left|\frac{\partial \varphi(x, y)}{\partial y}\right|
$$

由于遥感影像的 DN 值在空域上是离散的, 计算它的梯度模值就需将公式 (2) 离散化. 经过研究, Roberts 算子、Sobel 算子和 Prewitt 算子相继被提出并用来计算离散化的梯度模值 ${ }^{[17]}$. 考虑到 Sobel 算子计算 


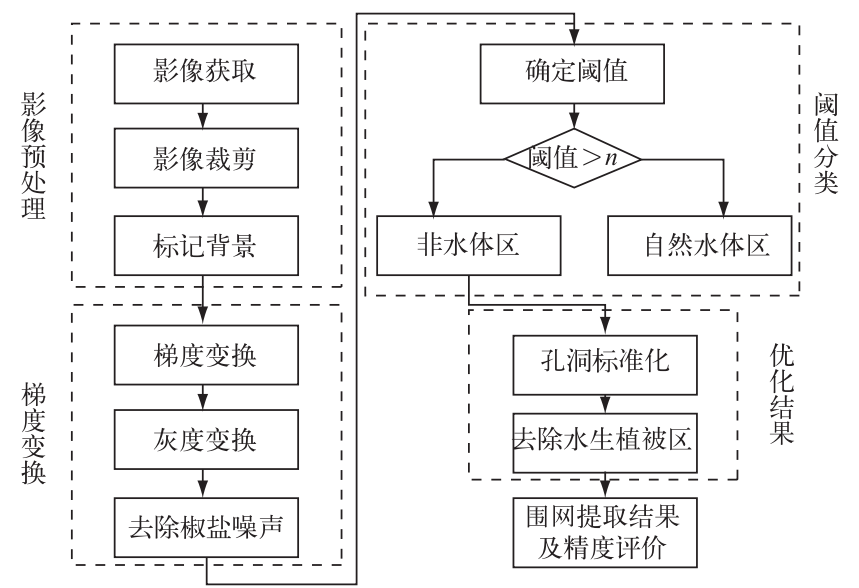

图 3 围网提取算法流程图

Fig.3 Flow chart for extracting enclosure culture area

后的结果相对于 Roberts 算子和 Prewitt 算子更精确及平滑 ${ }^{[18]}$, 本文使用 Sobel 算子. 根据算子 ${ }^{[10]}$ :

$$
\begin{aligned}
& \frac{\partial \varphi(i, j)}{\partial x}=(\varphi(i-1, j+1)+2 \varphi(i, j+1)+\varphi(i+1, j+1))-(\varphi(i-1, j-1)+ \\
& 2 \varphi(i, j-1)+\varphi(i+1, j-1)) \frac{\partial \varphi(i, j)}{\partial y}=(\varphi(i+1, j-1)+2 \varphi(i+1, j)+ \\
& \varphi(i+1, j+1))-(\varphi(i-1, j-1)+2 \varphi(i-1, j)+\varphi(i-1, j+1)) \\
& \text { 将式(3)代人式(2)可以得到: } \\
& \left|\nabla \varphi^{n}(i, j)\right| \cong \mid\left[\varphi^{n}(i+1, j-1)+2 \varphi^{n}(i+1, j)+\varphi^{n}(i+1, j+1)\right]-\left[\varphi^{n}(i-1, j-1)+\right. \\
& \left.2 \varphi^{n}(i-1, j)+\varphi^{n}(i-1, j+1)\right]|+|\left[\varphi^{n}(i-1, j+1)+2 \varphi^{n}(i, j+1)+\right. \\
& \left.\varphi^{n}(i+1, j+1)\right]-\left[\varphi^{n}(i-1, j-1)+2 \varphi^{n}(i, j-1)+\varphi^{n}(i+1, j-1)\right] \mid
\end{aligned}
$$

式中, $n$ 表示波段; $\varphi$ 表示影像波段的 DN 值; $i, j$ 分别为影像的行列数.

当 $n=4$ 时, 变化率可较好地识别出围网和自然水体 (图 2), 因此, 通过对第 4 波段进行以上梯度变换计 算,其结果如图 4.



图 4 梯度变换结果

Fig.4 Result of gradient transform 
统计梯度变换后的影像灰度值, 由统计结果可知 $0.001 \sim 0.150$ 区间的灰度值占比较高, 为增强特征利于 分类, 选用幂率函数中的根变换进行灰度变换 ${ }^{[17]}$. 此外, 自然情况下湖面浪花的镜面反射给梯度变换后的 新影像引人 “椒盐” 噪声 (图 4). 为了提高围网提取的可靠性, 本文采用中值滤波器去除椒盐噪声 ${ }^{[19]}$, 且中 值滤波器的模板大小根据噪声的空间密度而定 ${ }^{[20]}$.

\section{3 围网区提取及结果优化}

在进行灰度变换和去噪后, 进行围网区國值的确定, 具体方法为: 参考目视解译的结果, 在不同的区域 分别选取具有代表性和典型性的围网样本作为训练样本, 共选取 20 个样本, 并保证每个样本至少包含 10 个 围网网格, 然后计算样本的数学期望将其作为围网提取國值, 为 0.8 . 通过该國值将围网区全部提取, 结果如 图 5A. 但由于水生植被与水体在近红外的灰度值差异也较大, 因此, 此时提取的围网区还混有部分水生植 被区. 通过比较水生植被区和围网区的影像特征, 发现围网区区域内有孔洞, 且空间上孔洞分布存在周期 性, 但大小随空间分布没有规律 (图 5A). 为了剔除水生植被区, 找出孔洞是关键, 而标准大小的孔洞比随意 大小的孔洞更易识别. 根据围网的形状特征的统计结果, 发现围网孔洞大小基本介于 $4 * 4$ 像元和 $15 * 20$ 像元之间, 且频率最高的孔洞大小为 $12 * 18$ 像元. 为此, 本算法先逐行扫描围网区寻找间隔在 $4 \sim 15$ 像元之 间的围网网格, 并将这些网格通过填充 (像元灰度值设置为 1 ) 或挖去 (像元灰度值设置为 0 ) 的方式变成横 向间隔 12 像元的格子, 然后再逐列扫描寻找间隔在 4 20 像元之间的围网网格, 并以同样的方式将它们变 成纵向间隔 18 像元的格子. 这样就可以将其规格范围内的孔洞全部转变为 $12 * 18$ 像元大小 (图 5B). 通过 对围网区的像元个数进行统计, 可知阳澄湖围网区宽度基本都大于 $180 \mathrm{~m}$ 即 3 个孔洞周期, 因此本算法将篮 选出的孔洞周期数大于 3 的区域作为围网区, 结果如图 5C. 最后通过将空洞处灰度值设置为 1 , 将提取区域 的孔洞填满 (图 5D).
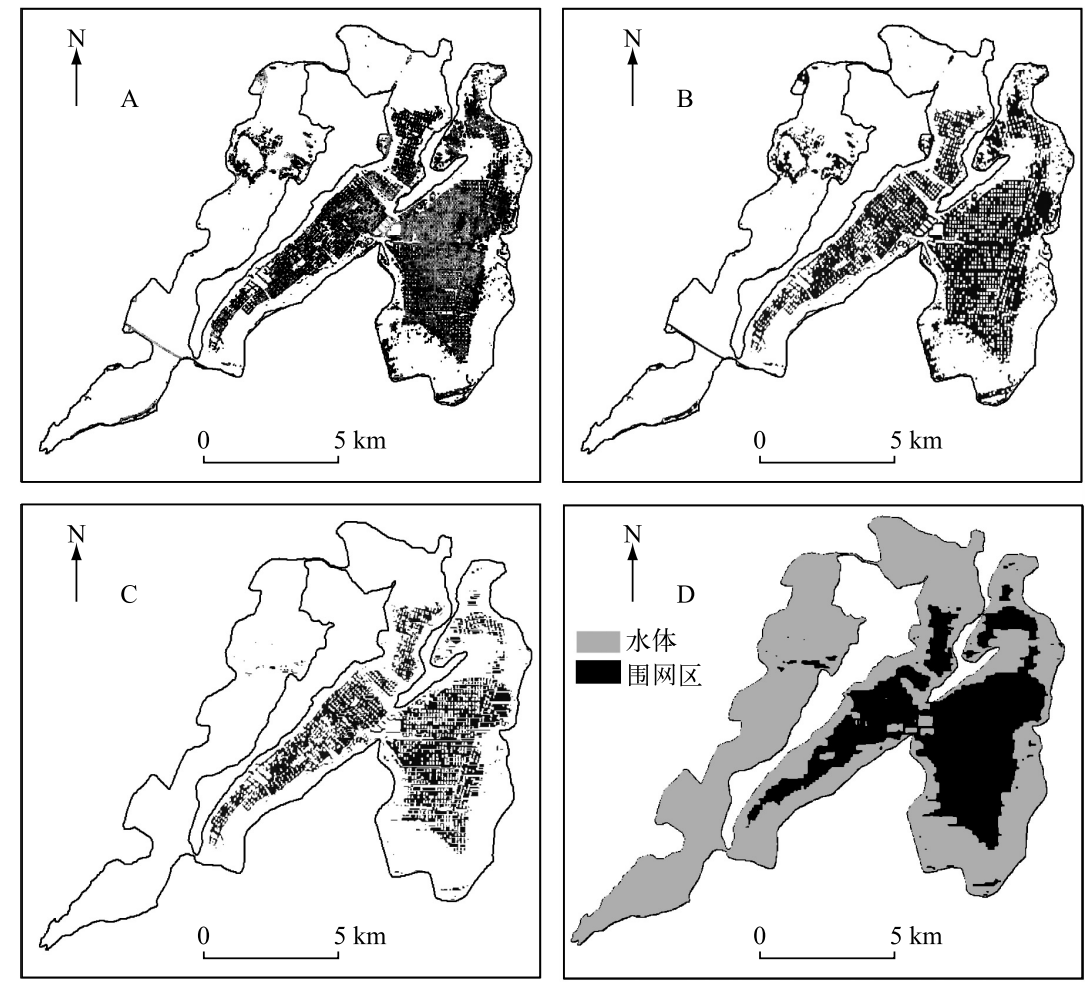

图 5 围网初步提取结果 $(A)$ 、围网孔洞标准化结果 $(B) 、$

去除水生植被区结果 $(\mathrm{C})$ 和围网最终提取结果 $(\mathrm{D})$

Fig.5 Initial extraction(A), standardizing hole(B), aquatic vegetation elimination( $\mathrm{C}$ ) and final extraction(D) maps 


\section{3 精度评价}

采用人工解译的围网区作为参考标准, 对本论文算法提取的围网区进行精度验证. 具体方法为: 将算法 提取的围网区和人工解译的围网区转变成矢量图层, 假设算法提取的围网区为集合 A, 人工解译的围网区为 集合 $B$, 则正确分类的围网区 $C=A \cap B$,漏分的围网区 $O=B-C$, 错分的围网区 $E=A-C$ ( 图 6), 分别计算 $A$ 、 $\mathrm{B} 、 \mathrm{C} 、 \mathrm{O} 、 \mathrm{E}$ 的面积,并根据以下公式计算漏分误差、错分精度和总体精度 ${ }^{[21]}$ :

$$
\begin{gathered}
p_{\mathrm{o}}=\frac{S_{\mathrm{O}}}{S_{\mathrm{B}}} \times 100 \% \\
p_{\mathrm{e}}=\frac{S_{\mathrm{E}}}{S_{\mathrm{A}}} \times 100 \% \\
p_{\mathrm{c}}=\frac{S_{\mathrm{C}}+S_{\mathrm{W}}}{S}=\frac{S_{\mathrm{C}}+S-S_{\mathrm{A}}-S_{0}}{S} \times 100 \%
\end{gathered}
$$

式中, $p_{\mathrm{o}}$ 为漏分误差, $p_{\mathrm{e}}$ 为错分误差, $p_{\mathrm{c}}$ 为总体精度, $S_{\mathrm{O}}$ 为漏分的面积, $S_{\mathrm{E}}$ 为错分的围网面积, $S_{\mathrm{C}}$ 为正确分 类的围网面积, $S_{\mathrm{W}}$ 为正确分类的水体面积, $S_{\mathrm{A}}$ 为算法提取的围网面积, $S_{\mathrm{B}}$ 为人工解译的围网面积, $S$ 为湖 泊总面积.

基于以上验证方法得到的精度评价结果如表 1. 该算法对围网区的提取精度为 $90.66 \%$, 错分精度为 $17.95 \%$, 漏分误差为 $15.35 \%$.
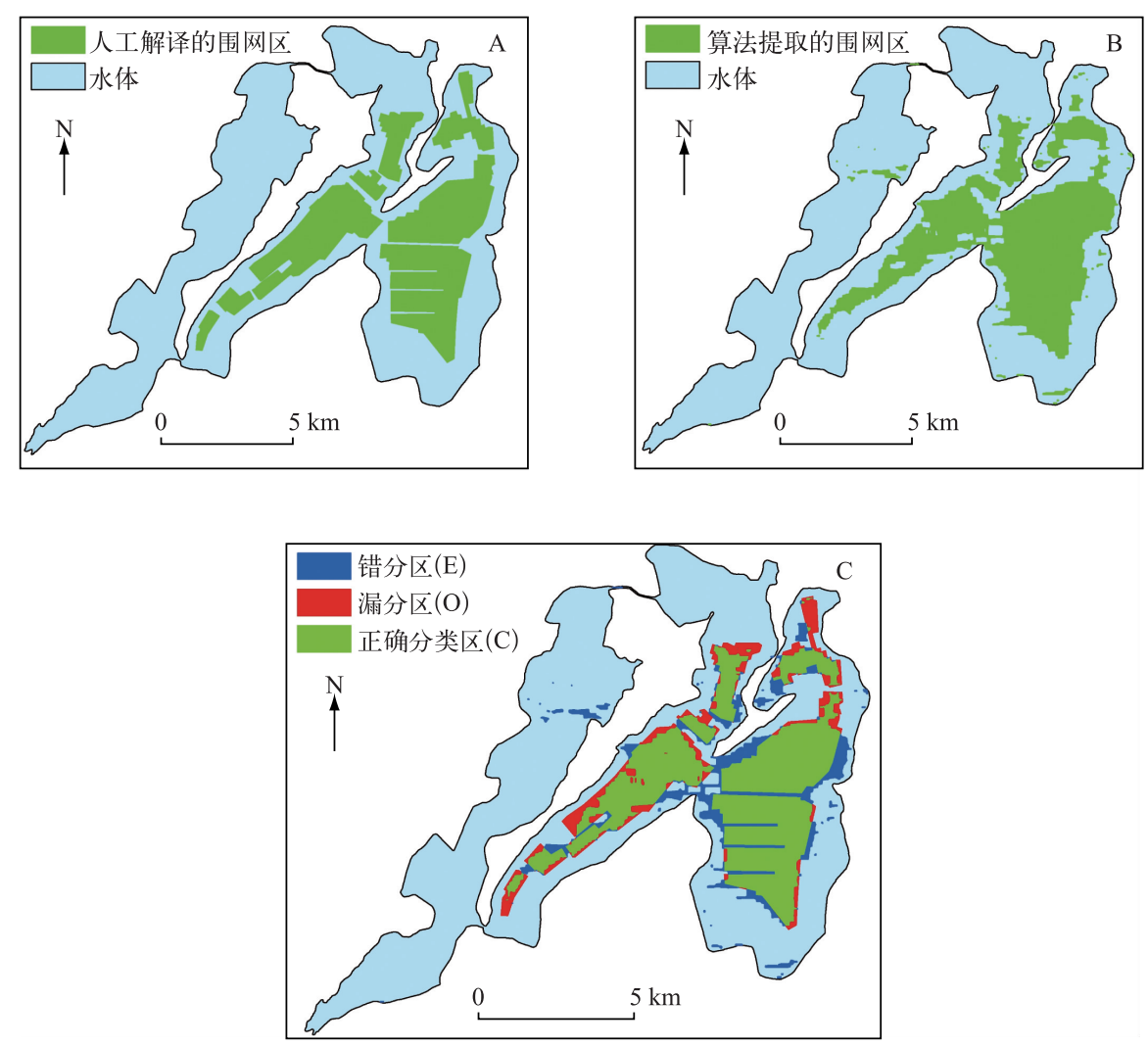

图 6 人工解译的围网区 $(A)$ 、算法提取的围网区 $(B)$ 和两者的比较结果 $(\mathrm{C})$

Fig.6 Classification maps using manual interpretation (A), algorithmic method (B), and their overlay intersecting map $(\mathrm{C})$ 
表 1 围网提取精度评价

Tab.1 Accuracy evaluation for extracting enclosure

\begin{tabular}{lcccc}
\hline 类型 & $\begin{array}{c}\text { 错分面积 } \\
\mathrm{km}^{2}\end{array}$ & $\begin{array}{c}\text { 漏分面积 } \\
\mathrm{km}^{2}\end{array}$ & $\begin{array}{c}\text { 人工解译的面积 } \\
\mathrm{km}^{2}\end{array}$ & $\begin{array}{c}\text { 算法提取的面积 } \\
\mathrm{km}^{2}\end{array}$ \\
\hline 自然水面 & 5.53 & 4.58 & 78.48 & 77.53 \\
围网养殖区 & 4.58 & 5.53 & 29.87 & 30.81 \\
\hline
\end{tabular}

\section{4 结论与讨论}

基于 ZY-3 卫星影像, 根据围网区与非围网区在影像上的光谱空间变化特征, 利用梯度变换提出一种围 网区遥感识别方法, 并利用该方法对 2015 年阳澄湖的围网区进行提取, 并以人工目视解译结果作为标准, 对提取结果进行验证和精度评价. 结果表明: 该方法对围网区的提取精度达到 $90.66 \%$, 基本满足实际应用 需求.

本文以阳澄湖为研究区提出了围网提取方法, 且取得较高的识别精度. 理论上, 该算法具有普适性, 可 用于其他浅水湖泊的围网提取,但考虑到不同湖泊的水环境因素差异较大,该算法能否准确提取其它的浅 水湖泊 (如滆湖、长荡湖等) 的围网区还有待进一步验证. 此外, 该算法是基于高分影像 ZY-3 为数据源发展 的, 理论上该算法可以用于任何卫星影像提取浅水湖泊围网, 但受空间和光谱分辨率的限制, 该算法是否适 用于其他卫星影像 (如更低分辨率的 Landsat 和 HJ-CCD 等) 提取浅水湖泊围网区, 精度如何等还有待进一 步研究. 同时,该算法依然存在误差, 理论上很多因素均会造成精度损失,如样本的选取方式、样本数量的确 定和阈值的计算等,如何分析不同因素对精度的影响进而提高提取精度还有待进一步研究.

\section{5 参考文献}

[ 1 ] Han Xiangzhen, Li Enhua, Yuan Longyi et al. The effects of enclosure aquaculture on aquatic vegetation and surface sediment resuspension. Hubei Agricultural Sciences, 2007, 46(4) : 556-558. [韩祥珍, 厄恩华, 袁龙义等. 围网养殖对水 生植被和沉积物再悬浮的影响. 湖北农业科学, 2007, 46(4) : 556-558.]

[ 2 ] Wang Xiumei, Song Youkun. Enclosure aquatic culture in lakes and the protection of water environment. Pollution Control Technology, 2006, 19(6): 3-4. [王秀梅, 宋友坤. 湖泊围网养殖与水环境保护. 污染防治技术, 2006, 19(6): 3-4. ]

[ 3 ] Ji Haiting, Xie Dong, Zhou Hengjie et al. Advances in ecological research on epiphytic community of submerged macrophytes. J Lake Sci, 2013, 25(2) : 163-170. DOI : 10.18307/2013.0201. [ 纪海婷, 谢冬, 周恒杰等. 沉水植物附植生 物群落生态学研究进展. 湖泊科学, 2013, 25(2) : 163-170.]

[ 4 ] Shen Fang, Kuang Dingbo. Remote sensing investigation and analysis for water resources utilization and its dynamic change of representing mid-or small lake groups in Taihu drainage area. Journal of Remote Sensing, 2003, 7(3): 221-226. [沈 芳, 匡定波. 太湖流域典型中小湖群水资源利用及动态变化的遥感调查与分析. 遥感学报, 2003, 7(3): 221-226.]

[ 5 ] Ma Yanjuan, Zhao Dongling, Wang Ruimei et al. Offshore aquatic farming areas extraction method based on ASTER data. Transactions of the Chinese Society of Agricultural Engineering, 2010, 26(S2): 120-124. [马艳娟, 赵冬玲, 王瑞梅等. 基于 ASTER 数据的近海水产养殖区提取方法. 农业工程学报, 2010, 26(S2)：120-124.]

[ 6 ] Zhu Changming, Luo Jiancheng, Shen Zhanfeng et al. Extract enclosure culture in coastal waters based on high spatial resolution remoter sensing image. Journal of Dalian Maritime University, 2011, 37(3): 66-69. [朱长明, 骆剑承, 沈占锋 等. 高分辨率遥感影像近海养殖区自动识别方法. 大连海事大学学报: 自然科学版, 2011, 37(3): 66-69.]

[ 7 ] Lu Yewei, Li Qiangzi, Du Xin et al. A method of coastal aquaculture area automatic extraction with high spatial resolution images. Remote Sensing Technology and Application, 2015, 30(3): 486-494. [卢业伟, 李强子, 杜金等. 基于高分辨 率影像的近海养殖区的一种自动提取方法. 遥感技术与应用, 2015, 30(3) : 486-494.]

[ 8 ] Li Junjie, He Longhua, Dai Jinfang et al. Extract enclosure culture in lakes based on remote sensing image texture information. J Lake Sci, 2006, 18(4) : 337-342. DOI : 10.18307/2006.0403. [李俊杰, 何隆华, 戴锦芳等. 基于遥感影像纹 
理信息的湖泊围网养殖区提取. 湖泊科学, 2006, 18(4) : 337-342.]

[ 9 ] Wang Jing, Gao Junfeng. Extraction of enclosure culture in Gehu Lake based on correspondence analysis. Journal of Remote Sensing, 2008, 12(5): 716-723. [王静, 高俊峰. 基于对应分析的湖泊围网养殖范围提取. 遥感学报, 2008, $12(5): 716-723$.

[10] Yuan Chunlan, Xiong Zonglong, Zhou Xuehua et al. Study of infrared image edge detection based on Sobel operator. Laser \& Infrared, 2009, 39(1) : 85-87. [袁春兰, 熊宗龙, 周雪花等. 基于 Sobel 算子的图像边缘检测研究. 激光与红外, $2009,39(1): 85-87$.

[11] He Chunhua, Zhang Xuefei, Hu Yingchun. A study on improved algorithm for Sobel on image edge detection. Optical Technique, 2012, 38(3) : 323-327. [ 何春华, 张雪飞, 胡迎春. 基于改进 Sobel 算子的边缘检测算法的研究. 光学 技术, $2012, \mathbf{3 8}(3)$ : 323-327.]

[12] Li Cancan, Sun Changhui, Wang Jing et al. Extraction of leaf vein based on improved Sobel algorithm and hue information. Transaction of the Chinese Society of Agricultural Engineering, 2011, 27(7) : 196-199. [李灿灿, 孙长辉, 王静等. 基于 改进的 Sobel 算子和色调信息的叶脉提取方法. 农业工程学报, 2011, 27(7) : 196-199.]

[13] Gabriel T, Vijay C, Tsai SS et al. Fast computation of rotation-invariant image features by an approximate radial gradient transform. IEEE Transactions on Image Processing. A Publication of the IEEE Signal Processing Society, 2013, 22( 8) : 2970-2982.

[14] Wang Jian, Liu Xiao, Wang Guohui. Multi-sensor image fusion algorithm based on gradient transform. Journal of Chongqing University of Technology, 2012, 26(10) : 62-65.

[15] Wang Sumin ed. Lakes of China. Beijing: Sciences Press, 1998: 360. [王苏民. 中国湖泊志. 北京: 科学出版社, 1998: 360.]

[16] Duan Ruiling, Li Qingxiang, Li Yuhe. Summary of image edge detection. Optical Technique, 2005, 31(3): 415-419. [段 瑞玲, 李庆祥, 李玉和. 图像边缘检测方法研究综述. 光学技术, 2005, 31(3): 415-419.]

[17] Gonzalez RC. Digital image processing. Beijing: Publishing House of Electronics Industry, 2006.

[18] Zhai Jun, Gan Lan. The application of grads Hough transformation in circle detection. Journal of East China Jiaotong University, 2007, 24(1) : 101-104. [翟钧, 甘岗. 梯度 Hough 变换在圆检测中的应用. 华东交通大学学报, 2007, 24 (1) : 101-104.]

[19] Zhao Gaochang, Zhang Lei, Wu Fengbo. Application of improved median filtering algorithm to image de-noising. Journal of Applied Optics, 2011, 32(4): 678-682. [赵高长, 张否, 武风波. 改进的中值滤波算法在图像去噪中的应用. 应用 光学, $2011,32(4): 678-682$.

[20] Zhang Heng, Lei Zhihui, Ding Xiaohua. An improved method of median filter. Journal of Image and Graphics, 2004,9 (4) : 408-411. [张恒, 雷志辉, 丁晓华.一种改进的中值滤波算法. 中国图象图形学报, 2004, 9(4) : 408-411.]

[21] Zhao Yingshi ed. Analysis and principle of remote sensing applications. Beijing: Sciences Press, 1998: 217. [ 赵英时. 遥 感应用分析原理与方法. 北京:科学出版社, 1998: 217.] 